Accepted after revision: February 5, 2010

\title{
Morphological and Extracellular Matrix Changes following Vocal Fold Injury in Mice
}

\author{
Masaru Yamashita Diane M. Bless Nathan V. Welham \\ Division of Otolaryngology - Head and Neck Surgery, Department of Surgery, University of Wisconsin School of \\ Medicine and Public Health, Madison, Wisc., USA
}

\section{Key Words}

Scarring $\cdot$ Tissue repair $\cdot$ Extracellular matrix $\cdot$ Vocal fold injury

\begin{abstract}
Mouse experimental models are commonly utilized tools in biomedical research but remain underrepresented in vocal fold biology, presumably due to the small size of the larynx and limited description of the anatomical, cellular and extracellular composition of the vocal folds. In this study, we provide a whole-mount serial section-based histological description of vocal fold morphology of wild-type FVB strain mice, alongside a histological and immunohistochemical (IHC)-based quantitative analysis of extracellular matrix (ECM) alteration 1, 7, 14, 28, 42 and 56 days following unilateral vocal fold injury. IHC was specific for procollagen type I, collagen type I, collagen type III, collagen type IV, elastin, decorin, fibronectin and hyaluronic acid binding protein 2 . The histological description confirmed the presence of a laryngeal alar structural complex in the mouse, which appears to be a morphological feature unique to rodents. The lamina propria appeared uniform without evidence of a distinct layer structure as has been reported in larger animals and humans. Time-dependent alterations in vocal fold morphology, ECM organization and ECM protein/glycoconjugate
\end{abstract}

abundance were observed in injured vocal folds compared to control. The presence of a mature scar was observed between 28 and 42 days postinjury. Morphological and ECM changes following vocal fold injury in the mouse were generally consistent with those reported in other animal models, particularly the rat, although wound repair in the mouse appears to occur at a faster rate.

Copyright $\odot 2010$ S. Karger AG, Basel

\section{Introduction}

The vocal fold is a unique layered structure consisting of epithelium, lamina propria (LP) and the thyroarytenoid (TA) muscle [Kurita et al., 1983]. The LP layer contains a rich extracellular matrix (ECM) that underpins vocal fold tissue viscoelasticity. The abundance and distribution of proteins and glycans within this ECM support vocal fold biomechanical performance for phonation, and their disruption is often detrimental to the voice [Gray et al., 1999, 2000]. Significant ECM disruption in the context of vocal fold scar is an intractable pathologi-

Presented at the 130th Annual Meeting of the American Laryngological Association, Phoenix, Ariz., USA, May 28-29, 2009.

\section{KARGER}

두 2010 S. Karger AG, Basel

Fax +41613061234 E-Mail karger@karger.ch www.karger.com www.karger.com/cto
Dr. Nathan V. Welham

Division of Otolaryngology - Head and Neck Surgery, Department of Surgery

University of Wisconsin School of Medicine and Public Health

K4/723 CSC, 600 Highland Avenue, Madison, WI 53792 (USA)

Tel. +1 608263 0121, Fax +1 608252 0939, E-Mail welham@surgery.wisc.edu 


\begin{tabular}{ll}
\hline Abbreviations used in this paper \\
\hline AC & arytenoid cartilage \\
ACA & alar cricoarytenoid muscle \\
ACo & anterior commissure \\
CC & cricoid cartilage \\
DAPI & $4^{\prime}$,6-diamidino-2-phenylindole dihydrochloride \\
ECM & extracellular matrix \\
EVG & elastica-van Gieson \\
Gla & subglottal gland \\
HA & hyaluronic acid \\
HABP2 & hyaluronic acid binding protein 2 \\
H\&E & hematoxylin and eosin \\
IHC & immunohistochemistry \\
LAC & laryngeal alar cartilage \\
LCA & lateral cricoarytenoid muscle \\
LP & lamina propria \\
L-TA & lateral thyroarytenoid muscle \\
M-TA & medial thyroarytenoid muscle \\
RT & room temperature \\
TA & thyroarytenoid muscle \\
TC & thyroid cartilage \\
VF & vocal fold \\
\hline
\end{tabular}

cal condition characterized by severe dysphonia and voice handicap [Hirano, 2005; Hansen and Thibeault, 2006; Welham et al., 2007]. Because of the large variability in scarring severity among clinical patients [Hirano et al., 2009], and researchers' inability to systematically control variables thought to impact treatment outcome, animal models are critical to progress in this area. In laryngeal research, animal model selection has generally been driven by a desire to work with a vocal fold structure and ECM constitution as close to that of humans as possible. While no animal model provides an ideal representation of the human vocal fold [Kurita et al., 1983], different species hold unique advantages for particular experimental applications.

Tateya et al. [2005] developed a rat surgical injury model to study vocal fold scarring outcomes, based on the argument that rats are ubiquitous in biomedical research and have a trilayered vocal fold LP, relatively short life span, and lower experimental costs than larger animals. Rats are also amenable to gene knock-out; however, as the technology to achieve knock-out in rats is dependent on induced mutagenesis and progeny screening, relatively few knock-out models are available [Zan et al., 2003; Smits et al., 2006; Cotroneo et al., 2007; van Boxtel et al., 2008].

Morphological/ECM Changes following Vocal Fold Injury in Mice
In contrast to rats, gene-manipulated (i.e. knock-out, knock-in, knock-down) mouse models are commonly utilized tools for studying the role of a specific gene and/ or gene-mediated pathway in a disease of interest. However, mouse experimental models have had limited use in vocal fold research to date, primarily due to the small size of the larynx and limited description of the anatomical, cellular and extracellular composition of the vocal folds. We recently reported an endoscopic surgical methodology for creating vocal fold injuries in FVB strain mice [Yamashita et al., 2009]. In order to take full advantage of this methodology using gene-manipulated models, it is first necessary to have a comprehensive understanding of the normal morphology and ECM changes associated with scar formation in wild-type mice. In this study, we used whole-mount serial sections to extend previous work describing the gross anatomy of the naïve mouse larynx [Thomas et al., 2009], and examined the alteration in the abundance of key ECM constituents in the LP up to 56 days following unilateral vocal fold injury.

\section{Materials and Methods}

This study was performed in accordance with the PHS Policy on Humane Care and Use of Laboratory Animals and the Animal Welfare Act (7 USC, 2131 et seq.); the animal use protocol was approved by the Institutional Animal Care and Use Committee (IACUC) of the University of Wisconsin-Madison.

\section{Experimental Animals and Surgical Injury}

Twenty-eight male FVB strain mice $(26.2 \pm 1.2 \mathrm{~g}$; Harlan Sprague-Dawley, Indianapolis, Ind., USA) were used in this study. Animals were divided into 7 experimental groups (4 animals/ group). Six groups underwent a unilateral (right-sided) vocal fold injury procedure followed by sacrifice and laryngeal tissue harvest $1,7,14,28,42$ and 56 days postinjury; one group was retained as a noninjury control condition.

Unilateral vocal fold injuries were created as previously described [Yamashita et al., 2009]. Briefly, mice underwent anesthesia induction with $2-3 \%$ isoflurane followed by intraperitoneal injection of a cocktail containing $50 \mathrm{mg} / \mathrm{kg}$ ketamine hydrochloride $(\mathrm{HCl}), 4 \mathrm{mg} / \mathrm{kg}$ xylazine $\mathrm{HCl}$ and $0.05 \mathrm{mg} / \mathrm{kg}$ atropine sulfate. Additional xylazine $\mathrm{HCl}$ was administered topically to the larynx and hypopharynx to prevent reflexive glottal closure during the surgical procedure. Vocal fold exposure was achieved using a custom-fabricated wire laryngoscope and tissue injury was performed under endoscopic guidance using a series of customfabricated microinstruments. A right-sided vocal fold injury, consisting of complete removal of the membranous vocal fold mucosa with exposure of the TA muscle, was successfully created in all experimental animals. None of the mice demonstrated physical distress, dyspnea or stridor during postsurgical observation. 

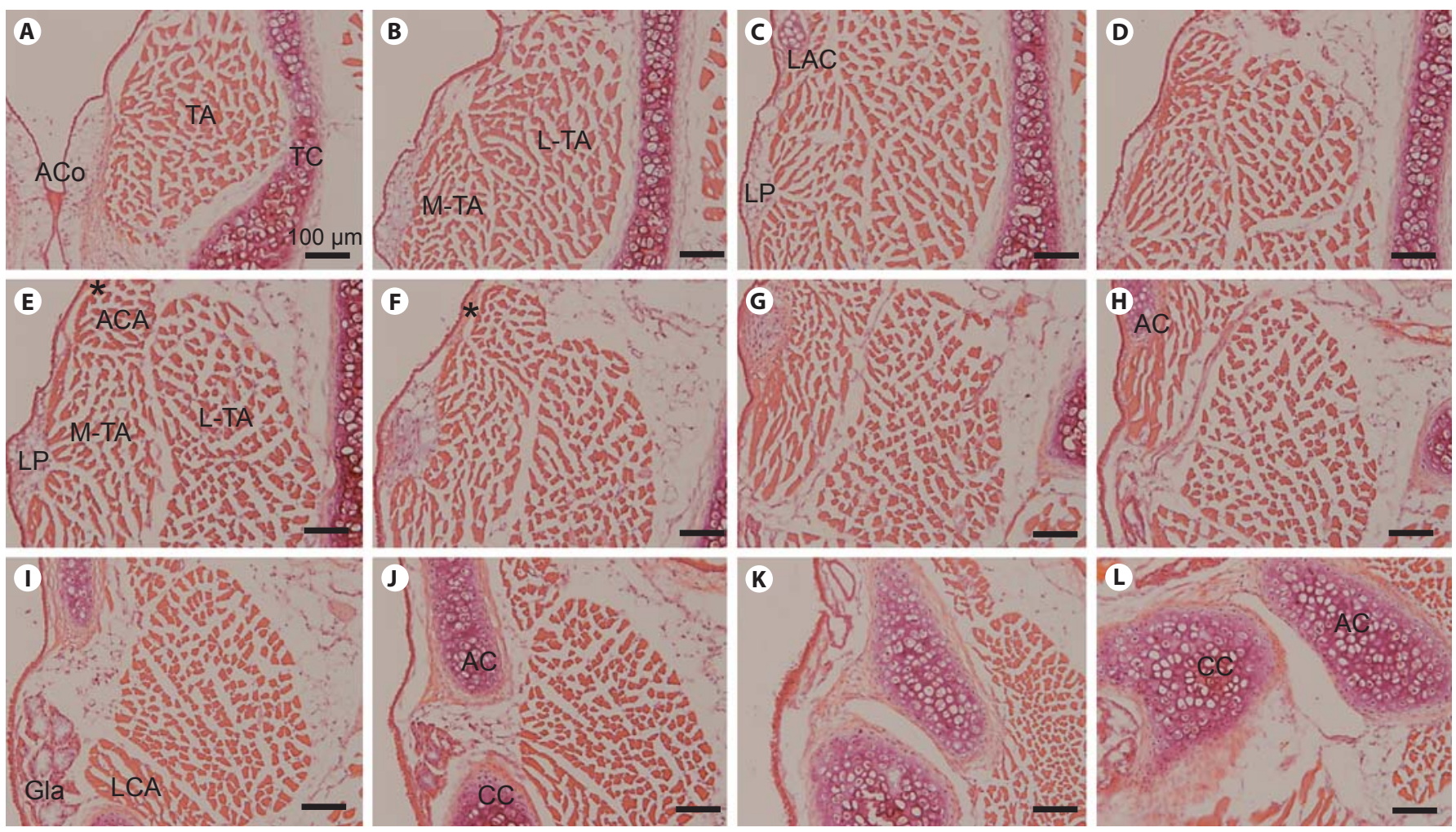

Fig. 1. Representative $H \& E$-stained images of serial whole-mount coronal sections from a wild-type FVB control mouse larynx. AL Panels are ordered from anterior to posterior; the distance between each panel is approximately $120 \mu \mathrm{m}$. AC = Arytenoid cartilage; $\mathrm{ACA}=$ alar cricoarytenoid muscle; $\mathrm{ACo}=$ anterior commissure; $\mathrm{CC}=$ cricoid cartilage; $\mathrm{Gla}=$ subglottal gland; $\mathrm{LAC}=$

laryngeal alar cartilage; LCA = lateral cricoarytenoid muscle; $\mathrm{LP}=$ lamina propria; L-TA = lateral thyroarytenoid muscle; MTA = medial thyroarytenoid muscle; TA = thyroarytenoid muscle; TC = thyroid cartilage. Asterisk indicates ligament-like structure extending from the LAC to the AC. Scale bar: $100 \mu \mathrm{m}$.

Tissue Harvest and Slide Preparation

Animals were euthanized by $\mathrm{CO}_{2}$ asphyxiation. Larynges were removed en bloc and dehydrated overnight at $4^{\circ} \mathrm{C}$ in phosphatebuffered saline containing $25 \%$ sucrose. Next, larynges were embedded in optimal cutting temperature compound (Tissue-Tek, Sakura Finetech, Tokyo, Japan), snap-frozen using acetone and dry ice, and stored at $-80^{\circ} \mathrm{C}$ until further use.

Serial $7-\mu \mathrm{m}$ cryosections were prepared using a cryostat (CM-3050 S, Leica, Wetzlar, Germany). One larynx per experimental group was sectioned in the axial plane, and the remaining three larynges were sectioned in the coronal plane. Sections were air-dried and stored at $-20^{\circ} \mathrm{C}$ prior to staining.

\section{Immunohistochemistry and Histology}

We employed fluorescent immunohistochemistry (IHC) followed by hematoxylin and eosin (H\&E) staining to evaluate ECM protein abundance and scar formation at successive time points following vocal fold injury. IHC was performed for procollagen type I, collagen type I, collagen type III, collagen type IV, elastin, decorin, fibronectin and hyaluronic acid binding protein 2 (HABP2). These proteins and glycoconjugates were selected as they are known vocal fold ECM constituents and have been shown

to undergo modulation during wound healing and scar formation in humans and other animal models [Thibeault et al., 2002; Rousseau et al., 2003; Tateya et al., 2005; Hirano et al., 2009]. Two midmembranous vocal fold coronal sections (corresponding to the anterior-posterior level shown in fig. 1C, D) were selected for each immunostain from each larynx. Routine H\&E staining of coronal sections from a normal uninjured mouse and axial sections from control and injured vocal folds of injured mice at each time point were performed to evaluate general vocal fold morphology and structural alteration associated with tissue repair and scar formation.

Sections intended for IHC were fixed with $4 \%$ paraformaldehyde for $4 \mathrm{~min}$ at room temperature (RT) and treated with 0.5\% Triton X-100 (Sigma-Aldrich, St. Louis, Mo., USA) in blocking solution (1:10; BUF029, Serotec, Raleigh, N.C., USA) for $15 \mathrm{~min}$ at RT. Image-iT FX signal enhancer (Invitrogen, Carlsbad, Calif., USA) was applied for $30 \mathrm{~min}$ at RT followed by blocking with the above-mentioned blocking solution for $10 \mathrm{~min}$ at RT. Primary antibodies were applied for $1 \mathrm{~h}$ at RT. Relevant secondary antibodies were also applied for $1 \mathrm{~h}$ at RT. 4',6Diamidino-2-phenylindole dihydrochloride (2 $\mu \mathrm{g} / \mathrm{ml}$ DAPI; MP Biomedicals, Santa Ana, Calif., USA) was applied for $20 \mathrm{~min}$ 
as a nuclear stain. Slides were washed 3 times, 5 min per wash, between each step.

The primary antibodies used in this study were rabbit anticollagen type I (1:100; ab34710, Abcam, Cambridge, Mass., USA), rabbit anti-collagen type III (1:50; 600-401-105, Rockland, Gilbertsville, Pa., USA), rabbit anti-collagen type IV (1:80; ab19808, Abcam), goat anti-decorin $(15 \mu \mathrm{g} / \mathrm{ml}$; AF1060, R\&D Systems, Minneapolis, Minn., USA), goat anti-elastin (1:50; sc-17580, Santa Cruz Biotechnology, Santa Cruz, Calif., USA), rabbit anti-fibronectin (1:250; LSL-LB-1027, Cosmo Bio, Tokyo, Japan), rabbit anti-HABP2 (1:200; LS-C15838, LifeSpan Biosciences, Seattle, Wash., USA), and goat anti-procollagen type I (1:50; sc-25973, Santa Cruz Biotechnology). The secondary antibodies used were Alexa Fluor 594 (1:400; anti-rabbit A-11012 and anti-goat A-11058, Invitrogen). Negative controls, for which samples were exposed to the secondary antibody in the absence of the primary antibody, revealed no immunostain.

\section{Image Capture and Analysis}

Stained sections were visualized and images were captured using a microscope with both bright field and fluorescent capabilities (E600, Nikon, Melville, N.Y., USA) connected to a digital microscopy camera (DP70, Olympus, Center Valley, Pa., USA). All images intended for quantitative analysis were captured with consistent exposure settings.

Image analysis software (MetaMorph 7.5, Molecular Devices, Sunnyvale, Calif., USA) was used to quantify the fluorescent positive area (in pixels) in the LP of each immunostained image. The threshold value for determining a positive signal was determined for each ECM protein/glycoconjugate immunostain according to its negative control and background signal strength. This threshold was then used consistently across all samples. The LP area was defined as deep to the epithelium-basement membrane complex (medial boundary) and superficial to the TA muscle (lateral boundary). Superior and inferior boundaries were identified by narrowing of the LP alongside widening of the adjacent supra- or subglottis. Images from adjacent $\mathrm{H} \& \mathrm{E}$-stained coronal sections were used to confirm LP area in cases where the boundary between the LP and TA muscle was unclear on immunostain (i.e. following injury). As reepithelialization was not observed 1 day following vocal fold injury, tissue overlying the TA muscle was selected as the region of interest in these cases. The number of positively stained pixels per LP was normalized to the entire LP area and expressed as a percentage. A total of 6 slides ( 3 animals, 2 coronal sections/animal) were analyzed per immunostain within each experimental group. Ten percent of immunostained sections were subjected to intrameasurer and intermeasurer reliability analyses.

\section{Statistical Analysis}

Image analysis data were analyzed using the Mann-WhitneyWilcoxon test. Normalized positive immunostain (percent positive pixels) was compared in the injured (right-sided) vocal fold LP at each postinjury time point against the control (i.e. day 0 ) condition; a parallel analysis was performed for the contralateral uninjured (left-sided) LP. Intra- and intermeasurer agreements were evaluated using Spearman's rank correlation coefficients $(\rho)$. An $\alpha$-level of 0.01 was used for all comparisons; all $p$ values were two-sided.

Morphological/ECM Changes following

Vocal Fold Injury in Mice

\section{Results}

\section{Anatomical Features of Mouse Vocal Folds}

We evaluated the general morphology of the naïve FVB strain mouse larynx using routine histological preparation and $\mathrm{H} \& \mathrm{E}$ staining. Figure 1 contains representative serial coronal sections, beginning at the anterior commissure (fig. 1A), which forms the anterior border of both vocal folds, and moving posteriorly. The right vocal fold is predominantly featured in this figure. The vocal fold and superior structures were covered by stratified squamous epithelium, which transitioned to pseudostratified ciliated epithelium in the subglottis. The TA muscle, which operates to shorten the vocal fold, was identified with medial and lateral compartments (M-TA and L-TA in fig. $1 \mathrm{~B}, \mathrm{E})$. The laryngeal alar cartilage (LAC), an anatomic feature unique to rodents, was located superior to the vocal fold (fig. 1C). From an endoscopic superior orientation, the shape of this cartilage resembled an inverted V. The alar cricoarytenoid muscle (ACA) (fig. 1E) was located superior to the TA and ran through the LAC to the arytenoid (AC in fig. 1H, J, L) and cricoid (CC in fig. 1J, $\mathrm{L})$ cartilages. The LP was located in the region between the M-TA and epithelium (fig. 1C, E). An eosinophilic ligament-like structure (asterisk in fig. 1E, F) was identified extending from the LAC to the AC.

\section{Alteration of Matrix Constituents following Vocal \\ Fold Injury}

We induced a unilateral vocal fold surgical injury and evaluated change in the abundance of ECM constituents procollagen type I, collagen type I, collagen type III, collagen type IV, elastin, decorin, fibronectin and HABP2 over time. IHC and quantitative image analysis revealed significant alteration in ECM organization and abundance at $1,7,14,28,42$ and 56 days postinjury compared to uninjured control (fig. 2, 3). Intrameasurer $\rho$ was 0.94 ; intermeasurer $\rho$ was 0.81 .

All ECM constituents were detected in the naïve vocal fold LP; however, procollagen type I (fig. 2A) and collagen type IV (fig. 2D) demonstrated a relatively weak immunostain under this condition (procollagen I is a collagen type I precursor and is therefore expected to be most abundant during periods of active matrix remodeling, collagen type IV is primarily a basement membrane protein and is therefore expected to be sparsely distributed in the naïve LP). Following vocal fold injury, procollagen type I abundance increased significantly at day 1 only (fig. 2A). Collagen type I (fig. 2B) and collagen type IV (fig. 2D) exhibited similar patterns of decreased abun-

Cells Tissues Organs 2010;192:262-271 


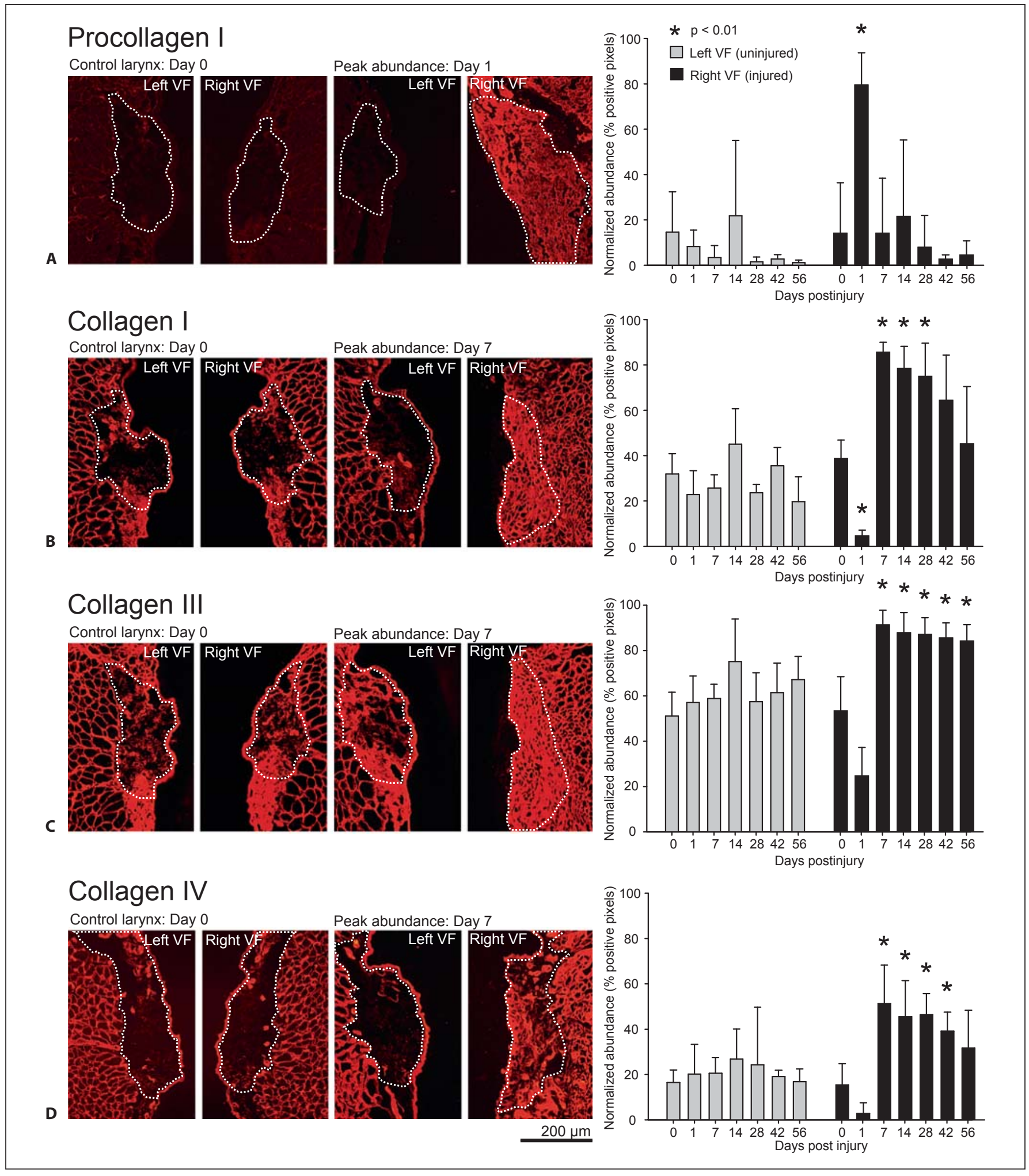

Fig. 2. Representative IHC images and quantitative analysis of procollagen type I (A), collagen type I (B), collagen type III (C), and collagen type IV (D) in uninjured control and injured vocal fold LP over time. Normalized abundance is expressed with re- spect to total LP area (indicated by a white dashed line). Error bars represent standard deviations and asterisks denote statistically significant differences $(\mathrm{p}<0.01)$ from the control group (day 0 ). $\mathrm{VF}=$ Vocal fold. Scale bar: $200 \mu \mathrm{m}$. 


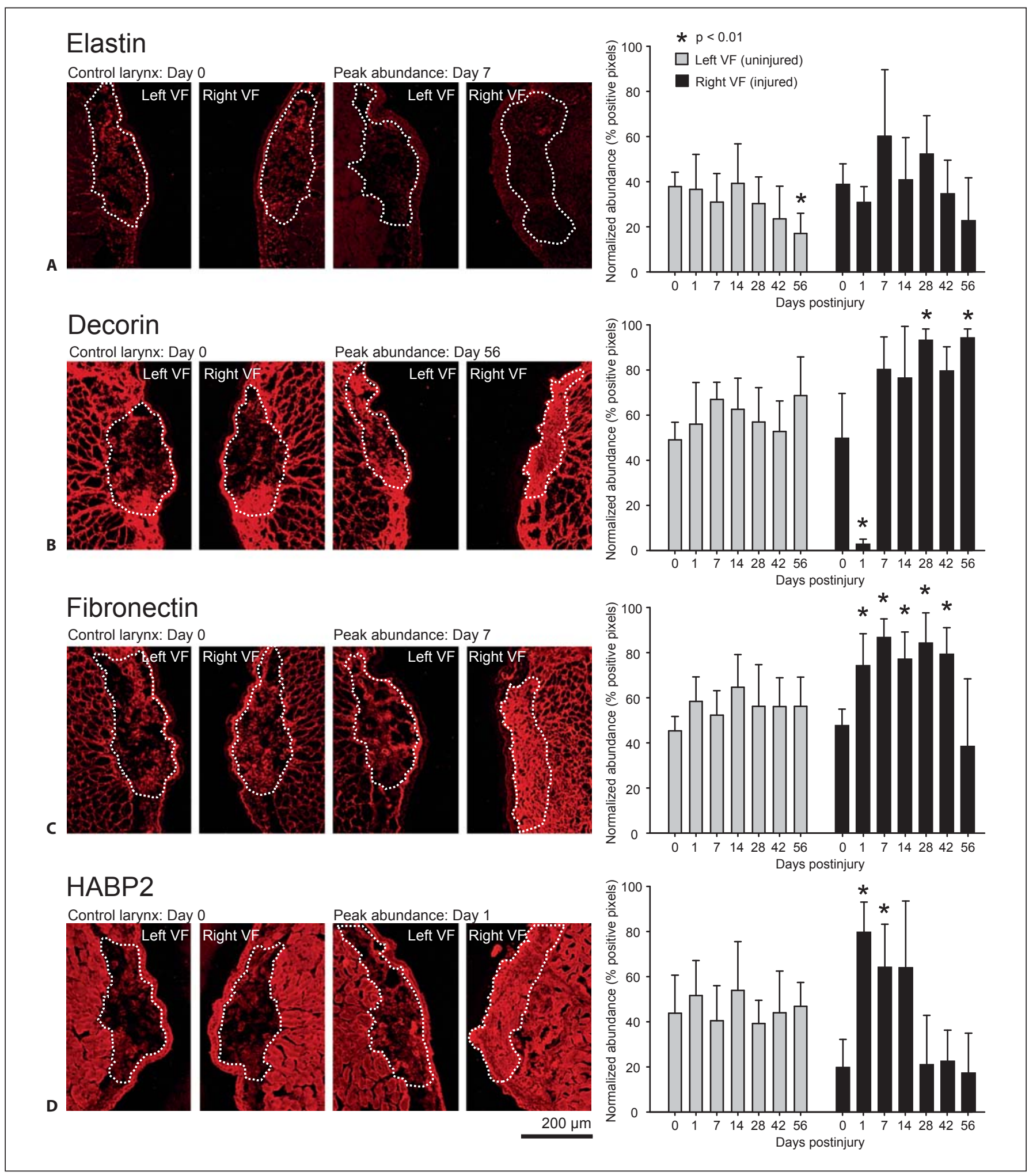

Fig. 3. Representative IHC images and quantitative analysis of elastin (A), decorin (B), fibronectin (C), and HABP2 (D) in uninjured control and injured vocal fold LP over time. Normalized abundance is expressed with respect to total LP area (indicated by a white dashed line). Error bars represent standard deviations and asterisks denote statistically significant differences $(\mathrm{p}<$ 0.01 ) from the control group (day 0$) . V F=$ Vocal fold. Scale bar: $200 \mu \mathrm{m}$. 

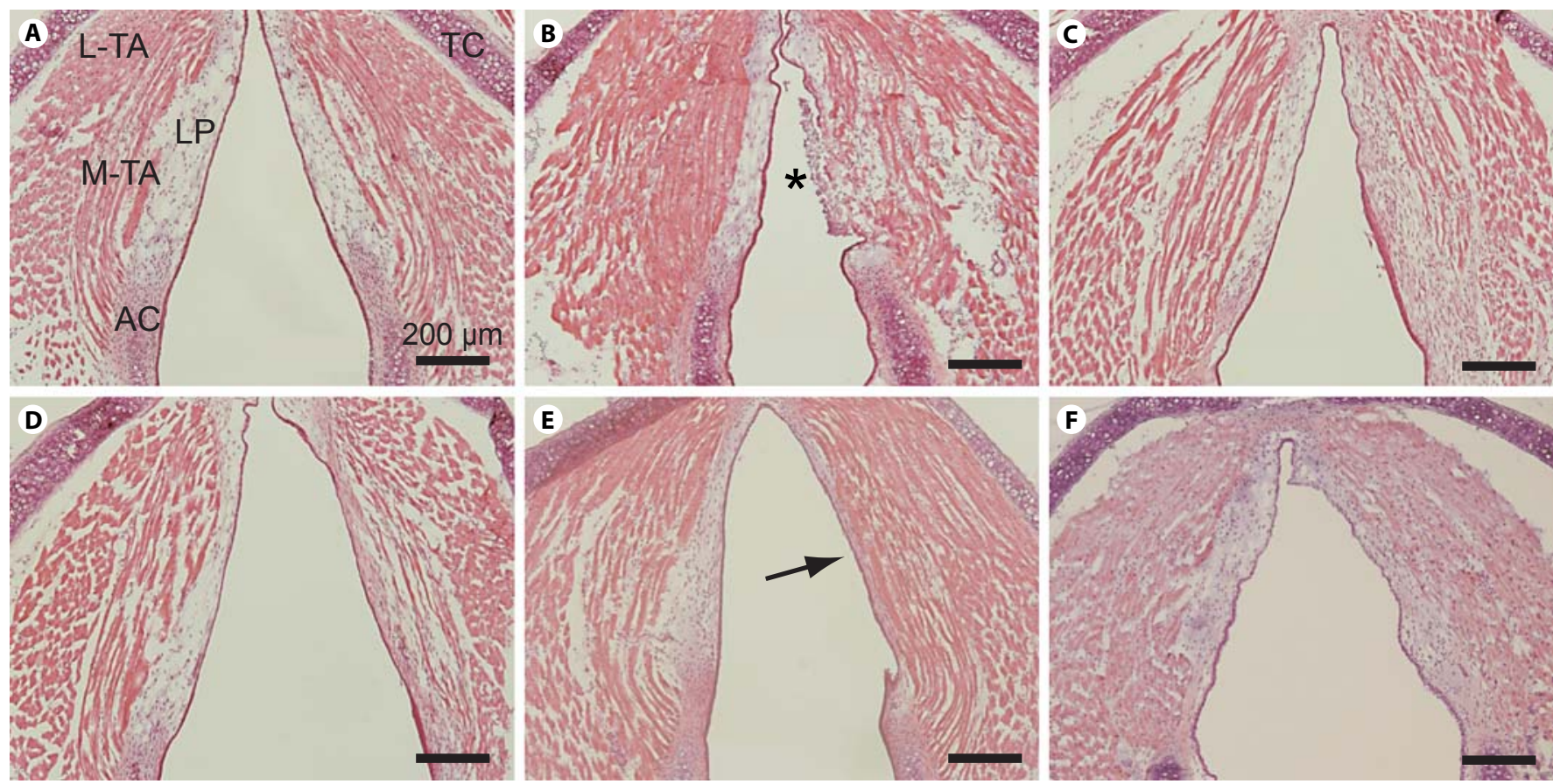

Fig. 4. Representative H\&E-stained images of whole-mount axial sections from an uninjured control mouse larynx and unilaterally injured mice larynges at 1, 7, 14, 28 and 42 days postinjury. A Uninjured control. B One day postinjury. C Seven days postinjury. D Fourteen days postinjury. E Twenty-eight days postinjury. Arrow indicates dense eosinophilic region and LP contraction. F Forty-two days postinjury. H\&E-stained whole-mount coronal sections from additional mice at each time point revealed comparable histopathology (data not shown). AC = Arytenoid cartilage; LP = lamina propria; L-TA = lateral thyroarytenoid muscle; $\mathrm{M}-\mathrm{TA}=$ medial thyroarytenoid muscle; $\mathrm{TC}=$ thyroid cartilage . Asterisk indicates fibrin clot and infiltration of inflammatory cells. Scale bar: $200 \mu \mathrm{m}$. dance at day 1 compared to control, maximum abundance at day 7 , and gradually decreasing abundance over the remaining time points. Collagen type III (fig. 2C) and decorin (fig. 3B) exhibited a pattern of decreased abundance on day 1 , followed by a sustained increase in abundance beginning on day 7. Elastin abundance was variable across all time points with no significant differences noted (fig. 3A). Fibronectin demonstrated increased abundance between days 1 and 42 (fig. 3C). HABP2 exhibited increased abundance between days 1 and 14 (fig. 3D).

\section{Morphological Changes following Vocal Fold Injury}

We evaluated morphological changes following vocal fold injury using routine histological preparation and $\mathrm{H} \& \mathrm{E}$ staining. Figure 4 contains representative $\mathrm{H} \& \mathrm{E}-$ stained whole-mount axial sections of a naïve control larynx and larynges 1, 7, 14, 28 and 42 days following unilateral vocal fold injury. Consistent with the coronal sections presented in figure 1 , the naïve control sample (fig. 4A) was characterized by a relatively simple LP structure. One day postinjury, the injured vocal fold was characterized by a fibrin clot, inflammatory cell infiltration and absent epithelium (fig. 4B). Reepithelialization occurred by 7 days postinjury (fig. 4C). By 28 days postinjury, the injured LP had contracted and appeared densely eosinophilic (fig. 4E), consistent with mature scar formation. H\&E-stained whole-mount coronal sections from additional mice at each time point revealed comparable histopathology (data not shown).

\section{Discussion}

Inagi et al. [1988] provided the first comprehensive description of rat laryngeal anatomy. They identified and named the LAC, which is not present in larger animals and humans, and provided a detailed account of the rat intrinsic laryngeal muscles. Tateya et al. [2005, 2006b] extended this work by introducing a vocal fold injury and 
scar model in the rat, which has been adopted by several groups in the investigation of ECM alteration during vocal fold wound healing. Laryngeal research using a mouse model is rare and to date has been primarily limited to nonsurgical studies [Renne et al., 1992; Renne and Gideon, 2006; Abdelkafy et al., 2007; Marques et al., 2007; Hamamoto et al., 2008; Nishio et al., 2008; Thomas et al., 2008,2009 ], presumably due to the inherent challenges of working with such small tissue samples and limited knowledge regarding normal morphology. These limitations have delayed the adoption of genetically manipulated mouse models in laryngeal research; however, recent advances indicate that the application and surgical manipulation of these models are feasible. We recently reported a methodology for endoscopic laryngeal surgery in FVB strain mice [Yamashita et al., 2009], and Thomas et al. [2009] recently described gross laryngeal cartilage and muscle structure in C57/BL6J strain mice. This study expands upon these previous reports by providing a whole-mount serial section-based histological description of vocal fold morphology of wild-type FVB strain mice, alongside IHC-based quantitative analysis of ECM alteration up to 56 days following unilateral vocal fold injury.

Similar to rats, mice have an LAC and a pair of ACAs, which course from the LAC to the CC and AC (fig. 1E). They also have an eosinophilic ligament-like structure extending from LAC to the AC (fig. 1F). The functional significance of this alar structural complex in rodents is unknown; however, based on its anatomical position, it may function to protect the airway during swallowing by shortening the distance between each AC and the epiglottis. Our histology and IHC data suggest that the mouse LP is relatively uniform without evidence of a distinct layer structure as has been reported in larger animals and humans [Kurita et al., 1983].

We employed fluorescent IHC to measure the abundance of eight ECM constituents in naïve and injured vocal folds over time. Procollagen type I is a precursor of collagen type I and therefore indicates new collagen type I synthesis. Collagen type I is a primary fibrous protein in connective tissue and is the most abundant collagen subtype in the human body. It demonstrates preferential localization to the most superficial and deep regions of the human LP and is believed to provide tensile strength during vocal fold oscillation [Tateya et al., 2006a]. Collagen type III is the most abundant and uniformly distributed collagen subtype in the vocal fold LP [Tateya et al., 2006a]. It forms a reticular fibrous network and plays a role in wound healing in the early stages following in- jury [Stephens and Thomas, 2002]. Collagen type IV is a nonfibrous protein and is highly abundant in the basement membrane region [Gray et al., 1994; Tateya et al., 2006a]. Our observation of reduced collagen type IV abundance 1 day postinjury is consistent with epithelial disruption induced by our surgical procedure, whereas the high level of collagen type IV 7 days postinjury is consistent with reepithelialization. Elastin is a fibrous protein and a key component of elastic fiber. It confers elasticity to the vocal fold LP and is therefore critical to biomechanical tissue performance [Gray et al., 2000]. The antibody employed in this study reacts to both tropoelastin, which is a precursor of elastin, and mature elastin. Decorin is a proteoglycan and a modulator of collagen fibrillogenesis [Reed and Iozzo, 2002]. Elevated decorin levels postinjury, as observed in our study, are associated with scar formation via regulation of the transforming growth factor- $\beta$ pathway; in contrast, reduced decorin has been associated with scarless wound healing [Beanes et al., 2001]. Fibronectin is a glycoprotein and functions as an adhesion molecule for cell-to-cell and cell-to-ECM interaction [Ruoslahti, 1988]. HABP2 binds to the glycosaminoglycan hyaluronic acid (HA) and infers HA abundance, which in turn is a primary contributor to vocal fold LP viscosity [Gray et al., 1999]. Each of these ECM constituents was detected in the mouse LP and the majority demonstrated alteration in abundance over time in response to tissue injury. This suggests that even though a distinct LP layer structure is not present, the mouse is useful as a model for ECM modulation and scar formation in response to vocal fold injury.

Previous work addressing vocal fold ECM alteration and chronic scar formation has been reported in human patients, and canine, rabbit and rat animal models, using a variety of ECM detection methods. Data from human patients 3-13 months following vocal fold tissue resection suggest that while most patients show elevated collagen abundance, ECM modulation varies widely across individuals, possibly due to the extent of LP resection and heterogeneity in individual wound healing [Hirano et al., 2009]. Rousseau et al. [2003] introduced a canine scarring model and reported increased procollagen type I 2 months postinjury (IHC), increased and disorganized collagen 6 months postinjury [elastica-van Gieson (EVG) stain], decreased and disorganized elastic fibers at 2 and 6 months postinjury (EVG stain), and no change in HA at either time point (Alcian blue stain with hyaluronidase digestion control). Thibeault et al. [2002] utilized a rabbit model and reported increased procollagen type I (IHC), decreased but highly disorganized collagen (trichrome 
strain), decreased and shortened elastic fibers (EVG stain), and no change in HA (colloidal iron stain with hyaluronidase digestion control) 2 months postinjury. A follow-up study 6 months postinjury in the rabbit reported elevated collagen (EVG stain) and no change in procollagen, elastin and HA abundance compared to noninjured control [Rousseau et al., 2004]. In comparing our data to that generated in humans and larger animals, it is important to note that the temporal progression of wound healing and ECM remodeling events is known to differ between rodents and larger animals [Tateya et al., 2005, 2006b]. Additionally, the use of antibodies against specific protein/glycoconjugate subtypes in this study differs from the general histology staining procedures reported above. Regardless of these differences, we observed early upregulation of procollagen type I, followed by subsequent upregulation of collagen types I, III and IV, which is consistent with the general trend for procollagen and collagen reported in the canine and rabbit. Also comparable to previous reports, $\mathrm{HA}$ (inferred by HABP2) abundance at later postinjury time points was comparable to control in our dataset. Inconsistent with the canine and rabbit, we observed no significant alteration in elastin abundance. Finally, we observed elevated abundance of decorin and fibronectin postinjury, two ECM glycoconjugates that were not investigated in these earlier reports.

With the exception of HA, our findings in mice are generally consistent with data reported by Tateya et al. [2005] 14-84 days postinjury in a rat model. They observed increased collagen type I and III abundance at all postinjury time points (IHC); similar to our findings, collagen type I abundance tapered following an initial peak, whereas collagen type III abundance was sustained throughout the observation period. Fibronectin abundance increased at 14 and 28 days postinjury (IHC), and $\mathrm{HA}$ abundance decreased at all postinjury time points (Alcian blue stain with hyaluronidase digestion control). Our observation of initial HABP2 upregulation followed by a return to control levels is consistent with other reports showing that early accumulation of HA functions to stabilize the ECM, stimulate cell infiltration and migration and modulate fibrin degradation [Weigel et al., 1986; Chen, 2002]. Also consistent with our data and these reports, Thibeault et al. [2004] reported general downregulation of $\mathrm{HA}$ in the first 15 days following vocal fold injury in rabbits, but noted peak abundance comparable to uninjured control at 5 days postinjury.

We observed a fibrin clot and infiltration of inflammatory cells 1 day postinjury and reepithelialization by 7 days postinjury. These observations are consistent with our previous report [Yamashita et al., 2009] and suggest that vocal fold reepithelialization in mice occurs more rapidly than the 7-14 days reported in rats [Tateya et al., 2006b]. The LP appeared contracted and eosinophilic by 14 days and typical morphological features of scarred vocal folds were evident by 28 days postinjury. Together, our histological and IHC data suggest that it takes 28-42 days to form a mature scar in the mouse LP. These findings represent a useful comparative dataset for evaluating acute and chronic injury outcomes in genetically manipulated mice.

Our findings demonstrate that a mouse model is well suited to the study of ECM alteration associated with vocal fold injury and scar formation. Our previously reported surgical methodology was successful in creating an acute injury and chronic scar in the mouse that was characterized using conventional histology and IHC. Further, our findings suggest that data from other species cannot be used to determine the temporal progression of wound healing and ECM remodeling events in the mouse, because although the events observed in this study are generally consistent with those reported in other animal models (particularly rat), wound repair in the mouse occurs at a faster rate.

\section{Acknowledgements}

This study was supported by grant R01 DC004428 from the National Institute on Deafness and Other Communication Disorders. The first author was supported financially by a research fellowship gifted by the Uehara Memorial Foundation. We gratefully acknowledge technical assistance and consultation provided by Ronald McCabe, BSEE, image analysis assistance provided by Changying Ling, $\mathrm{PhD}$, and statistical consultation provided by Glen Leverson, $\mathrm{PhD}$ and Victoria Rajamanickam, MS.

References

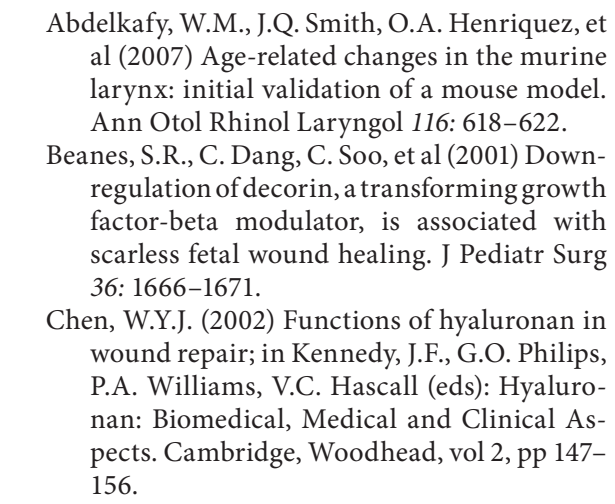

Yamashita/Bless/Welham 
Cotroneo, M.S., J.D. Haag, Y. Zan, et al (2007) Characterizing a rat Brca2 knockout model. Oncogene 26: 1626-1635.

Gray, S.D., S.S. Pignatari, P. Harding (1994) Morphologic ultrastructure of anchoring fibers in normal vocal fold basement membrane zone. J Voice 8: 48-52.

Gray, S.D., I.R. Titze, F. Alipour, T.H. Hammond (2000) Biomechanical and histologic observations of vocal fold fibrous proteins. Ann Otol Rhinol Laryngol 109: 77-85.

Gray, S.D., I.R. Titze, R. Chan, T.H. Hammond (1999) Vocal fold proteoglycans and their influence on biomechanics. Laryngoscope 109: $845-854$.

Hamamoto, T., M. Takumida, K. Hirakawa, S Takeno, T. Tatsukawa (2008) Localization of transient receptor potential channel vanilloid subfamilies in the mouse larynx. Acta Otolaryngol 128: 685-693.

-Hansen, J.K., S.L. Thibeault (2006) Current understanding and review of the literature: vocal fold scarring. J Voice 20: 110-120.

-Hirano, S. (2005) Current treatment of vocal fold scarring. Curr Opin Otolaryngol Head Neck Surg 13: 143-147.

-Hirano, S., S. Minamiguchi, M. Yamashita, T Ohno, S. Kanemaru, M. Kitamura (2009) Histologic characterization of human scarred vocal folds. J Voice 23: 399-407.

-Inagi, K., E. Schultz, C.N. Ford (1998) An anatomic study of the rat larynx: establishing the rat model for neuromuscular function. Otolaryngol Head Neck Surg 118: 74-81.

Kurita, S., K. Nagata, M. Hirano (1983) A comparative study of the layer structure of the vocal fold; in Bless, D.M., J.H. Abbs (eds): Vocal Fold Physiology: Contemporary Research and Clinical Issues. San Diego, College Hill Press, pp 3-21.
Marques, M.J., R. Ferretti, V.U. Vomero, E. Minatel, H.S. Neto (2007) Intrinsic laryngeal muscles are spared from myonecrosis in the mdx mouse model of Duchenne muscular dystrophy. Muscle Nerve 35: 349-353.

Nishio, T., H. Bando, H. Bamba, Y. Hisa, H. Okamura (2008) Circadian gene expression in the murine larynx. Auris Nasus Larynx 35: 539-544.

Reed, C.C., R.V. Iozzo (2002) The role of decorin in collagen fibrillogenesis and skin homeostasis. Glycoconj J 19: 249-255.

Renne, R.A., K.M. Gideon (2006) Types and patterns of response in the larynx following inhalation. Toxicol Pathol 34: 281-285.

Renne, R.A., K.M. Gideon, R.A. Miller, P.W. Mellick, S.L. Grumbein (1992) Histologic methods and interspecies variations in the laryngeal histology of F344/N rats and B6C3F1 mice. Toxicol Pathol 20: 44-51.

Rousseau, B., S. Hirano, R.W. Chan, et al (2004) Characterization of chronic vocal fold scarring in a rabbit model. J Voice 18: 116-124.

Rousseau, B., S. Hirano, T.D. Scheidt, et al (2003) Characterization of vocal fold scarring in a canine model. Laryngoscope 113: 620-627.

Ruoslahti, E. (1988) Fibronectin and its receptors. Annu Rev Biochem 57: 375-413.

Smits, B.M., J.B. Mudde, J. van de Belt, et al (2006) Generation of gene knockouts and mutant models in the laboratory rat by ENUdriven target-selected mutagenesis. Pharmacogenet Genomics 16: 159-169.

Stephens, P., D.W. Thomas (2002) The cellular proliferative phase of the wound repair process. J Wound Care 11: 253-261.

Tateya, T., I. Tateya, D.M. Bless (2006a) Collagen subtypes in human vocal folds. Ann Otol Rhinol Laryngol 115: 469-476.

Tateya, T., I. Tateya, J.H. Sohn, D.M. Bless (2005) Histologic characterization of rat vocal fold scarring. Ann Otol Rhinol Laryngol 114 183-191.
Tateya, T., I. Tateya, J.H. Sohn, D.M. Bless (2006b) Histological study of acute vocal fold injury in a rat model. Ann Otol Rhinol Laryngol 115: 285-292.

Thibeault, S.L., S.D. Gray, D.M. Bless, R.W. Chan, C.N. Ford (2002) Histologic and rheologic characterization of vocal fold scarring. J Voice 16: 96-104.

Thibeault, S.L., B. Rousseau, N.V. Welham, S. Hirano, D.M. Bless (2004) Hyaluronan levels in acute vocal fold scar. Laryngoscope 114: $760-764$

Thomas, L.B., G.L. Joseph, T.D. Adkins, F.H. Andrade, J.C. Stemple (2008) Laryngeal muscles are spared in the dystrophin deficient mdx mouse. J Speech Lang Hear Res 51: 586-595

Thomas, L.B., J.C. Stemple, R.D. Andreatta, F.H. Andrade (2009) Establishing a new animal model for the study of laryngeal biology and disease: an anatomic study of the mouse larynx. J Speech Lang Hear Res 52: 802-811.

van Boxtel, R., P.W. Toonen, M. Verheul, et al (2008) Improved generation of rat gene knockouts by target-selected mutagenesis in mismatch repair-deficient animals. BMC Genomics 9: 460.

Weigel, P.H., G.M. Fuller, R.D. LeBoeuf (1986) A model for the role of hyaluronic acid and fibrin in the early events during the inflammatory response and wound healing. J Theor Biol 119: 219-234.

Welham, N.V., S.H. Dailey, C.N. Ford, D.M. Bless (2007) Voice handicap evaluation of patients with pathologic sulcus vocalis. Ann Otol Rhinol Laryngol 116: 411-417.

Yamashita, M., D.M. Bless, N.V. Welham (2009) Surgical method to create vocal fold injuries in mice. Ann Otol Rhinol Laryngol 118: 131-138.

$\checkmark$ Zan, Y., J.D. Haag, K.S. Chen, et al (2003) Production of knockout rats using ENU mutagenesis and a yeast-based screening assay. Nat Biotechnol 21: 645-651. 